\title{
ROTORS FOR CELL STUDIES
}

\section{Subcellular Components}

Preparation and Fractionation. By G. D. Birnie and Sylvia M. Fox. Pp. viii + 173. (Butterworths: London, March 1969.) 60s.

The zonal rotor designed by Dr Anderson is a major development in the technology of centrifugation. These rotors are now commercially available, and this fact stimulated Dr Birnie and Miss Fox to organize a meeting to discuss some of the problems involved in the use of such rotors for the separation of subcellular components. The meeting was held in November 1967 and this volume contains the papers given at the meeting together with some of the discussion that ensued.

The main virtue of the zonal rotor is its large capacity which makes possible the preparation of much greater quantities of material than can be achieved in swinging bucket rotors. The use of the zonal rotor for isolating ribosomes and ribosomal subunits is described by Birnie, Fox and Harvey in the last chapter. Perhaps an even greater problem than the separation of subcellular components, however, is their release from the cell without damage and a good deal of the rest of the book is concerned with such matters.

Avis describes the pressure homogenization technique for the disruption of cells. In practice, this involves subjecting the cells to a very high pressure of nitrogen and then rapidly releasing the pressure. From the discussion that followed it is clear that we do not quite know how this procedure leads to the release of the organelles in an intact form, but it certainly appears to be a promising method. Roodyn surveys seventeen methods for the isolation of nuclei. He concludes that there is no ideal method for the isolation of uncontaminated nuclei suitable for metabolic and chemical studies. He pleads for the application of adequate criteria for the assessment of the purity and structural and metabolic integrity of the isolated nuclei. Chappell and Hansford describe the preparation of mitochondria from rat liver, heart and brain and from blow-flight muscle and yeast. They also discuss methods for the assessment of prepara. tions. Porteous contributes an original chapter on the isolation of microvilli from the epithelial cells of the intestine. The chapter by Tata on the preparation and properties of microsomal and submicrosomal fractions from animal cells is particularly valuable. This is a most difficult and complex arca, for the problem is not to isolate a particle such as a mitochondrion but to sub-fractionate pieces of a continuous mesh of membranes. While the chapter heading mentions animal cells, I think the reader should be warned that the material is virtually confined to liver and that the results cannot be applied directly to other tissues.

A rather different contribution is that by Flarnm, Birnstiel and Walker on the preparation and fractionation, and isolation of single strands, of DNA by isopycnic ultracentrifugation in fixed-angle rotors. The authors provide much valuable information on techniques which have no doubt been learnt the hard way, but they also explain the results on theoretical grounds wherever possible. Their advocacy of the fixed-angle rotor is very convincing and will come as a surprise to many.

A newcomer to this field is faced with a rather bewildering number of choices for the isolation of a component in which he is interested. This volume will be valuable in that the various contributors have considered these choices critically. Many of those who have previously worked in the field may have made their choice of method on largely empirical grounds and for these the authors provide new insight concerning the rationale behind the methods. The organizers and Measuring and Scientific Equipment Limited, who financed the symposium, are to be congratulated on making this useful volume available.

P. N. Campbelt

\section{BORDERLINE TYPE}

\section{The Biology of Euglena}

Edited by Dennis E. Buetow. Vol. 1: General Biology and Ultrastructure. Pp. xii +361 . (Academic Press: New York and London, December 1968.) 177s $4 d$.

Euglena, besides being a favourite "type" for elementary courses in biology, is an excellent subject for many kinds of experimental work and has been widely used in studies on nutrition, biochemistry and cytoplasmic inheritance. A graph given in this book shows an exponential increase, accurately maintained since 1953 with a doubling-time of a little over three years, in the number of papers published on the genus. Obviously it is useful to bring this copious information together in a comprehensive account and the authoritative articles contained in this volume, together with the biochemical contributions in the second volume, will be valued by teachers and research workers in many fields.

The taxonomy, phylogeny and evolution of the genus Euglena, topics that are also covered in a recent book by G. F. Leedale (reviewed in Nature, 217, 201; 1968), are discussed by L. P. Johnson, and its general ecology by J. B. Lackey. The surprising conclusion of a chapter on locomotive and motile response, by T. L. Jahn and E. C. Bovee, is that little is known of these in Euglena species themselves so that much of what is given is reasoning by analogy and some is purely speculative. Morphology and ultrastructure are well described and illustrated by $D$. E. Buetow and nuclear cytology and behaviour by G. F. Leedale. J. R. Cook's contribution on cultivation and growth will be particularly useful to those beginning experimental studies with Euglena. It is a pity that it is implied that the turbidostat continuous culture apparatus developed by Myers operates on the same principle as the chemostat. In the final chapter, B. W. Wilson and B. H. Levedahl make comparisons between the synthetic and division rates of Euglena and those of metazoan cells. Because Euglena is so often described as being on the border line between plant and animal, a comparison with plant cells as well would have been informative-plenty of suitable data for Chlorella are available. It is to be regretted also that a more satisfactory solution was not found to the difficult problem of comparing the metabolic patterns of different species. The allometric method, introduced long ago by Huxley for study of problems of relative growth, has been used with some success for this purpose and deserves to be more widely known.

It is clear that huge gaps still exist in our knowledge and that we are a long way from an integrated picture of even so well known an organism as Euglena. This is particularly evident in the account of the ecology, which, through no fault of the author, is largely qualitative and anecdotal in character. It is symptomatic that this chapter appears so early in the book. Ideally, precise explanation of the occurrence and behaviour of an organism in its natural environment should be one of the principal goals of laboratory studies and the chapter on ecology the culmination of, not the preamble to, those on physiology and biochemistry. It will be a step in this direction if investigators using Euglena heed the plea of Wilson and Levedahl that cell plus medium, rather than the cell itself, should be regarded the real unit of physiological activity of the growing culture. G. F. FoGG

\section{CHEMICAL ANALYSIS}

A Brief Introduction to Quantitative Chemical Analysis By Robert B. Fischer and Dennis G. Peters. Pp. xii + 537. (W. B. Saunders: Philadelphia and London, 1969.) $78 s 6 d$.

ONLY infrequently does the appearance of yet another 